\title{
Chemical development of preceramic polyvinylsilazane photoresist for ceramic patterning
}

\author{
Yi-He Li • Kuk-Ro Yoon • Xiao-Dong Li • \\ Dong-Pyo Kim
}

Published online: 4 December 2007

(C) Springer Science + Business Media, LLC 2007

The authors regret that one author, Kuk-Ro Yoon from the Department of Chemistry, Hannam University, Daejeon 305-811, Korea, was omitted from the article Chemical development of preceramic polyvinylsilazane photoresist for ceramic patterning.

The online version of the original article can be found at http://dx.doi. org/10.1007/s10832-007-9331-z.

Y.-H. Li $\cdot$ X.-D. Li

CFC key lab of National University of Defense Technology,

Changsha 410073 Hunan, People's Republic of China

Y.-H. Li • D.-P. Kim $(\bowtie)$

Department of Fine Chemical Engineering and Chemistry,

Chungnam National University,

Daejon 305-764, South Korea

e-mail:dpkim@cnu.ac.kr

\section{K.-R. Yoon}

Department of Chemistry,

Hannam University,

Daejeon 305-811, South Korea 